\title{
ACCESO A LAS TICS EN LOS ENTORNOS RURALES. APROXIMACIÓN DESDE LA PERSPECTIVA COMPARADA ENTRE ESPAÑA Y LA UNIÓN EUROPEA.
}

\section{ACCESS TO ICTS IN RURAL AREAS. APPROACH FROM THE COMPARATIVE PERSPECTIVE BETWEEN SPAIN AND THE EUROPEAN UNION.}

\section{Cristina Benlloch- Doménech ${ }^{1}$}

\section{RESUMEN}

El objetivo de este trabajo es analizar algunas de las diferencias de uso y acceso a las nuevas tecnologías entre los entornos rurales y urbanos, a partir de la encuesta de Equipamiento de TICs en forma comparada entre España y la Unión Europea, con las posibles desigualdades que esto pueda ocasionar para el desarrollo de la tecnologización de dichos entornos. Una de las conclusiones obtenidas es que cada vez las diferencias entre uno y otro contexto (el rural y el urbano) es menor, pero que la composición demográfica puede ser un factor clave para la mayor expansión de las TICs en los contextos rurales.

Palabras clave: Nuevas tecnologías, rural, urbano, vida telemática.

\section{ABSTRACT}

The objective of this work is to analyze some of the differences in use and access to new technologies between rural and urban environments, based on the ICT Equipment survey in a comparative way between Spain and the European Union, with the possible inequalities that This may lead to the development of the technologization of these environments. One of the conclusions obtained is that the differences between one context and the other (rural and urban) are less and less, but that demographic composition may be a key factor for the greater expansion of ICTs in rural contexts.

Keywords: New technologies, rural, urban, telematic life

\footnotetext{
${ }^{1}$ Doctorado de las Ciencias Sociales de las Relaciones laborales y de los recursos humanos. Afiliação: Universidad de Valencia- Espanha. ORCID: https://orcid.org/0000-0001-7802-4705 E-mail: cristina.benllo ch@uv.es
} 


\section{ACCESO A LAS TICS EN LOS ENTORNOS RURALES. APROXIMACIÓN DESDE LA PERSPECTIVA COMPARADA.}

\section{EL CONCEPTO DE LO RURAL Y APROXIMACIÓN A LAS TICS}

El concepto rural sugiere una elevada diversidad de imágenes (Hart et al., 2005). Se ha venido debatiendo sobre él desde el propio inicio de la sociología rural (SOROKIN Y ZIMMERMAN, 1929). Tanto es así que Hogart (1988) afirmaba que no había una definición de ruralidad. Además, se multiplica la complejidad cuando comprendemos que el concepto de ruralidad no es un universal científico; porque el uso del concepto nueva ruralidad adquiere dimensiones diferentes en Europa y América Latina, a pesar de estar atravesadas por las dinámicas que impone el capitalismo actual (TRPIN, 2005:4).

En la actualidad, este concepto también se encuentra en pleno debate, puesto que la sociología rural establece, como se verá, el límite entre lo rural y lo urbano en una diversidad de definiciones.

La definición de las unidades territoriales que se consideran rurales no es, por tanto, tan sencilla como se pueda pensar a priori, sino que se trata de un entramado complejo de características. Por ejemplo, el INE (Instituto Nacional de Estadística) considera que un municipio rural es aquel que tiene menos de dos mil habitantes; intermedio es aquel que tiene entre dos mil y diez mil habitantes; y ciudad, aquel que está por encima de los diez mil. Sin embargo, esta es solo una cuestión de números, aunque relevante a la hora de realizar los análisis ${ }^{2}$.

Los municipios rurales de este país muestran una elevada casuística que va de las poblaciones envejecidas sin posibilidad de regeneración social o reemplazo generacional a la ruralidad dinámica convertida en dormitorio para personas que trabajan en áreas urbanas y vuelven a dormir al municipio ${ }^{3}$ (CAMARERO ET AL., 2009).

Uno de los problemas de los contextos rurales es el hecho de que el peso demográfico recae sobre una generación a la que se está denominando la generación soporte. Se entiende por tal el conjunto de personas que se encuentran en el centro gravitacional de la pirámide de población, esto es, entre los treinta y los cincuenta años, y que en este caso está masculinizada

\footnotetext{
${ }^{2}$ Véase el glosario del censo en https://www.ine.es/censo_accesible/es/glosario.html disponible el 1 de agosto de 2020.

${ }^{3}$ Buscando la tranquilidad y la calidad medioambiental construida a partir de una cierta idealización de los rural, que contribuyen a una representación positiva de estos espacios que se conoce como el idilio rural (Camarero et al., 2009:10).
} 
(CAMARERO ET AL., 2009). Esta generación, por el peso que soporta, se denomina también generación sándwich, tal como la denominó Miller (1981), porque eran quienes estaban encargados de cuidar de sus mayores, pero también de sus hijos, de ahí el concepto de sándwich. Son, en realidad, las personas que mantienen la viabilidad de la pirámide, de ahí la importancia de que este colectivo esté presente en una sociedad determinada de forma suficiente, pues de ellos depende el futuro más inmediato, pero también el recorrido a largo plazo.

El punto de partida de este texto es analizar algunas de las diferencias de uso y acceso de las nuevas tecnologías entre los entornos rurales (atendiendo a las diferentes dimensiones de población) y las posibles desigualdades que esto pueda tener para el desarrollo de la tecnologización de dichos entornos, con la relevancia que tienen para el desarrollo de los contextos. Y más en un contexto como el que estamos viviendo, en el que las tecnologías han sido centrales en cierta medida para todos los aspectos de la vida cotidiana (desde el trabajo al estudio, incluso para la asistencia sanitaria a través de las nuevas tecnologías).

Nos estamos refiriendo a que durante la pandemia de la COVID-19, según los datos aportados por Eurofound (2020), más del $40 \%$ de las personas reportaron algún tipo de teletrabajo. Pero también los centros educativos de todos los niveles cerraron y la docencia pasó a ser en modalidad no presencial o telemática. Tanto el cuerpo docente como el estudiantil se vieron abocados a las clases a distancia o, en algunos casos, a una especie de enseñanza híbrida. Esto también ha afectado a las relaciones sociales, incluyendo las familiares y las de amistad. Existen estudios sobre las consecuencias de esta situación que analizan diferentes factores, entre los que se incluyen la clase social, la edad o la geografía, por ejemplo; $y$, sin embargo, no se ha llegado a tener en consideración, como punto de partida, el diferencial entre los entornos rurales y urbanos. Este trabajo trata de dar una humilde respuesta a estos problemas.

En este contexto consideramos que es importante analizar las posibilidades de acceso a las nuevas tecnologías en los entornos rurales. Así, el objetivo de partida del texto es analizar el potencial de los entornos rurales en relación a las nuevas tecnologías.

La metodología utilizada para ello es el análisis cruzado de los datos de población de diferentes organismos como ahora el Eurostat o el Banco Mundial con los datos de teletrabajo durante la pandemia de la COVID-19 con y la encuesta de uso de las TICs que se realiza en toda Europa. 


\section{LAS NUEVAS TECNOLOGÍAS, ENTORNOS RURALES DURANTE Y CRISIS SANITARIA DE LA COVID-19}

Con la COVID, las nuevas tecnologías han tomado un papel más central, si cabe, en nuestra vida cotidiana; aunque hace años que la vida digital - o el mundo online, the onlife world (HILDEBRANT, 2016: 45) - amenaza con ensombrecer la vida real. Y esto no solo en el tiempo en el que duró el confinamiento iniciado en marzo de 2020, sino que después del confinamiento muchas personas han seguido su trabajo y sus estudios de forma no presencial de manera intermitente.

Según datos de población del Banco Mundial, en el 2020 alrededor del 25\% de la población europea era rural; mientras que en España el porcentaje era del 19\%. Décadas atrás, en el año 1960 eran el $41 \%$ y el $43 \%$ respectivamente ${ }^{4}$.

La tasa de dependencia muestra el porcentaje de personas dependientes sobre las personas potencialmente independientes, es decir, entre los 16 y los 64 años, que son las personas en edad de trabajar. En los contextos eminentemente rurales se aprecia una mayor diferencia entre los hombres y las mujeres ${ }^{5}$ tanto en España como en Europa.

Entre las mujeres de más de 65 años en España el dato es del $50 \%$. Esta tasa de dependencia se muestra mucho mayor que entre los varones, donde la tasa es del $39 \%$. En la Europa de los 27 la tasa de dependencia de mayores de 65 no es tan elevada, pero sigue existiendo una brecha en lo que respecta al sexo, puesto que las mujeres presentan una mayor tasa de dependencia. Estos datos se comprenden mejor cuando se analizan las pirámides de población.

La población menor de 16 años está menos presente en las zonas españolas eminentemente rurales que en las europeas. La tasa de dependencia está equilibrada entre los niños y las niñas. Estos municipios tienen la media de edad más elevada de todos los municipios, por lo que la tasa de dependencia también es mayor a consecuencia del envejecimiento. Será interesante un análisis de los datos después de que la situación sanitaria se haya estabilizado.

\footnotetext{
${ }^{4}$ Datos obtenidos a partir de las estimaciones del Banco de Datos del Banco Mundial https://data.world bank.org/indicator/SP.RUR.TOTL.ZS?locations=EU-ES

${ }^{5}$ Se calcula la tasa de mujeres dependientes sobre mujeres. El cálculo de la tasa de dependencia se ha realizado desagregado por sexos para ver la diferencia que existe entre la distribución de edades de las mujeres y de los hombres. Se ha realizado con los datos de población en años quinquenales, por lo que se trata de una aproximación. Es útil para conocer el envejecimiento y la capacidad de reproducción de la sociedad a largo plazo. La población rural y la urbana es la que está compuesta por las zonas eminentemente urbanas o rurales, por lo que se analiza a través de los NUT3.
} 
TABLA 1. Tasa de dependencia en los municipios eminentemente rurales. Por sexos. Año 2020.

\begin{tabular}{|c|c|c|c|c|c|}
\hline & \\
\hline & & & Hombres & Mujeres & Ambos sexos \\
\hline \multirow{4}{*}{$\begin{array}{l}\text { Eminentemente } \\
\text { rural }\end{array}$} & Menos de 16 & \multirow[b]{2}{*}{ EU-27 } & $24 \%$ & $24 \%$ & $24 \%$ \\
\hline & Más de 65 & & $29 \%$ & $40 \%$ & $35 \%$ \\
\hline & Menos de 16 & \multirow[b]{2}{*}{ España } & $18 \%$ & $18 \%$ & $18 \%$ \\
\hline & Más de 65 & & $39 \%$ & $50 \%$ & $44 \%$ \\
\hline
\end{tabular}

Fuente. Elaboración propia a partir de datos de Eurostat ${ }^{6}$

En las zonas eminentemente urbanos la tasa de dependencia de las personas de más de 65 años es mucho menor que en los contextos eminentemente rurales; de la misma manera que ocurre con los menores de 16.

En este sentido, se puede concluir que mayor ruralidad mayor es la tasa de dependencia de las personas de más de 65 años, y la población está más envejecida; mientras que las cohortes menores de 16 años tienen un peso relativo mayor en los núcleos urbanos, lo que permite una mejor pervivencia a medio y largo plazo ${ }^{7}$. En esta línea de análisis también se desarrolla el informe de envejecimiento en Europa que apunta que existe una mayor predisposición de la población en edad avanzada a residir en los entornos más rurales.

TABLA 2. Tasa de dependencia en los municipios eminentemente urbanos. Por sexos. Año 2020.

\begin{tabular}{|c|c|c|c|c|c|}
\hline & & & Hombres & Mujeres & Ambos sexos \\
\hline \multirow{4}{*}{$\begin{array}{c}\text { Eminentemente } \\
\text { urbano }\end{array}$} & Menos de 16 & \multirow[b]{2}{*}{ EU-27 } & $24 \%$ & $23 \%$ & $23 \%$ \\
\hline & Más de 65 & & $26 \%$ & $34 \%$ & $30 \%$ \\
\hline & Menos de 16 & \multirow[b]{2}{*}{ España } & $23 \%$ & $22 \%$ & $23 \%$ \\
\hline & Más de 65 & & $24 \%$ & $32 \%$ & $28 \%$ \\
\hline
\end{tabular}

Fuente. Elaboración propia a partir de datos de Eurostat ${ }^{8}$

Esta aproximación a la composición demográfica de las zonas es importante también para comprender la relación de las personas que viven en estos contextos con las nuevas tecnologías; y con ellas las desigualdades que pueda haber, sobre todo, a la hora de afrontar

\footnotetext{
${ }^{6}$ Elaborado a partir de los datos de población de 1 de Enero de Eurostat por otras tipologías. Aproximación a partir de grupos quinquenales. Disponible en https://appsso.eurostat.ec.europa.eu/uni/show.do?datas et=urt_pjangrp3\&lang=en el 28 de septiembre de 2021

${ }^{7}$ De hecho, al analizar las medias de edad se comprueba que mientras que la media de edad española se situaba en 2018 aproximadamente en 43 años (42 para los hombres y 44 para las mujeres), en los municipios de menos de dos mil habitantes, la media de edad estaba en 49 años (48 para los hombres y 50 para las mujeres), un lustro por encima.

${ }^{8}$ Elaborado a partir de los datos de población de 1 de Enero Eurostat por otras tipologías. Aproximación a partir de grupos quinquenales. Disponible en https://appsso.eurostat.ec.europa.eu/nui/show.do?data set=urt_pjangrp3\&lang=en el 28 de septiembre de 2021.
} 
una situación como la actual en la que las TIC se han convertido en un eje de supervivencia en la pandemia (atención médica telefónica, trámites online, compra, trabajo, etc.). Por lo que resulta interesante analizar el punto de partida de ambos contextos, el rural (sobre todo, el de menos de dos mil habitantes) y el urbano (más de diez mil habitantes).

El acceso a los elementos de telecomunicaciones es fundamental para analizar la brecha tecnológica entre las diferentes unidades territoriales, es por ello por lo que se trabajará con los datos de la encuesta de TIC de 2018 en el que se preguntaba a la sociedad acerca de temas vinculados con las nuevas tecnologías como, por ejemplo, capacidad de acceso o usos ${ }^{9}$.

El acceso a las TIC no solo tiene relevancia para la participación ciudadana o para incrementar la visibilidad de entornos y colectivos sociales, sino también para aspectos como la economía o la comunicación, entre otros. Se ha comentado que con la implementación de las nuevas tecnologías en los contextos laborales se podría encontrar una vía para la repoblación, ya que se atraería a dichos espacios a personas que pudieran desarrollar su trabajo desde casa o con una menor prespecialidad, basada en lo estrictamente necesario. Esta pandemia ha puesto de manifiesto que muchas de las actividades laborales que estábamos desempeñando de forma presencial era posible realizarlas de forma telemática. En los medios de comunicación han aparecido noticias en los últimos meses acerca de la instalación de familias en pequeños municipios como efecto de los confinamientos y de la situación sanitaria, buscando unos contextos más amplios y menos densos socialmente ${ }^{10}$.

Este es un aspecto del que se viene hablando ya desde hace años. Así, Colom (2004) establecía las posibilidades que podrían tener las nuevas tecnologías para el desarrollo rural a través del teletrabajo. También se ha destacado su potencial para fijar a la población rural (FORONDA, 2004). Sin embargo, parece que este aspecto por sí mismo no desempeña un papel relevante para el mantenimiento y crecimiento poblacional de los núcleos rurales, sino que habría otros aspectos que reforzar para mejorar la percepción de las personas sobre los entornos rurales. En algunas zonas de Cataluña y Francia (por mencionar algunos ejemplos) se ha detectado desde hace años cómo personas procedentes de los entornos urbanos se iban

\footnotetext{
9 Esta encuesta nació en el año 2002. En ella se preguntan diversas cuestiones acerca no solo del equipamiento tecnológico del que disponen los hogares, sino también sobre el uso y la percepción de las nuevas tecnologías. Una de las ventajas que ofrece esta encuesta es el hecho de que se repita de forma anual y que esté armonizada en el contexto de Eurostat (González, 2013).

${ }^{10}$ En el trabajo de campo se observó que las personas que residían en los municipios tenían sensación de que en los pueblos se contagiaba menos la gente porque no se vivía tan hacinado (este trabajo se realizó mayoritariamente antes de la tercera ola). Sin embargo, los datos han mostrado que los pueblos tampoco han escapado de las consecuencias de la COVID.
} 
asentando en las zonas rurales buscando modelos de vida más tranquilos. Esto produjo algunos casos de gentrificació $n^{11}$ de estos entornos (BLANCO Y CANOVÉS, 2006). Esta es la situación que parece que se ha incrementado como consecuencia de la pandemia de la COVID-19 gracias, en parte, al teletrabajo.

Antes de la COVID-19, en España apenas el 3,2\% de los trabajadores había hecho algunas horas de teletrabajo; mientras que en el conjunto de la Unión Europea era del 9,9\%. Aunque en los últimos años, según las cifras oficiales de Eurostat, la tendencia era moderadamente al alza ${ }^{12}$.

Con la COVID-19, cuando se impusieron los confinamientos esta cifra se multiplicaría. En la encuesta realizada por Eurofound, al menos el $48 \%$ había respondido que había teletrabajado de algún modo durante la pandemia, un 34\% lo había hecho exclusivamente desde casa. Según esta misma fuente, en España esta cifra se situaba en torno al $40 \%$ de la fuerza de trabajo (Eurofound,2020).

Esto suponía un incremento sustancial con respecto de la situación anterior, pero también suponía adaptar los hogares a esta nueva situación, así como la forma en la que nos relacionábamos con la tecnología.

Además, las TIC podrían tener una relevancia en el acceso a las decisiones de la ciudadanía por parte de la población rural, puesto que estas podrían acercar a los ciudadanos a las decisiones políticas. En este sentido, también podría tener relevancia para frenar la despoblación rural, porque se podrían iniciar procesos de participación por los que dichos emplazamientos puedan mostrar su opinión en igualdad de condiciones que se puede hacer en las urbes. Sin embargo, es necesario tener en cuenta que la homogeneidad no es un elemento propio de dichos contextos, pero sí permitiría la interacción en la dispersión geográfica.

La revolución de internet es relativamente reciente si tenemos en cuenta el cambio que esta herramienta ha significado en la vida cotidiana de las personas, y aun así queda mucho recorrido por andar. En 1998, Marqués hablaba de cómo internet podría ser una revolución educativa. En este sentido, la escuela rural -o más bien los estudiantes rurales- podría beneficiarse de estas herramientas para acceder a recursos educativos que únicamente se encuentran disponibles en las ciudades por una cuestión de concentración poblacional. Todas estas teorías se pusieron a prueba durante el confinamiento con diferentes resultados.

\footnotetext{
${ }^{11}$ El término gentrificación nace en la década de los sesenta para explicar los procesos que se están viviendo en determinados barrios obreros en los que se produce un desplazamiento de la población como consecuencia del asentamiento en los mismos de personas de mayor poder adquisitivo (Garnier, 2017).

${ }^{12}$ Cifras extraídas del Boletín informativo del INE “El teletrabajo en España y la EU antes de la COVID-19".
} 
Además, es necesario tener en cuenta que el acceso a las nuevas tecnologías se produce de forma equitativa y segura. En este sentido, Jiménez (2016) realizó un estudio sobre las prácticas de participación en internet de las mujeres rurales andaluzas, y mostraba que existían diferencias entre los perfiles de mujeres, aunque su participación digital era más bien moderada.

\section{LA COMPLEJA RELACIÓN ENTRE RURALIDAD Y TICS}

El coste de acercar internet a las zonas remotas hace que sea complejo llevar a estos las herramientas necesarias para el acceso, pero en la actualidad existen posibilidades menos costosas como el 4G, por ejemplo. Es por ello por lo que se conjugan diferentes acciones para el acceso al mismo por parte de los contextos rurales (CARMONA Y MARTíNEZ, 2006). En lo que respecta al acceso a internet, hace apenas una década en las zonas rurales únicamente el $31 \%$ de los hogares disponían de acceso a internet. En la actualidad, en España el 91 \% de los mismos tiene acceso; esta cifra llega a más del $97 \%$ en el contexto urbano ${ }^{13}$.

En los últimos dos años, la España rural se ha situado por delante de la media de Europa y está en cifras similares a las de la media de las ciudades europeas, en las que en 2020 el $93 \%$ de los hogares disponían de acceso a internet de una forma u otra. Este elemento es muy importante en la situación actual si se tienen en cuenta el incremento del teletrabajo y las restricciones de movilidad.

GRÁFICA 1. Acceso a internet por tipo de zona. De 2007 a 2020. Por nivel territorial.

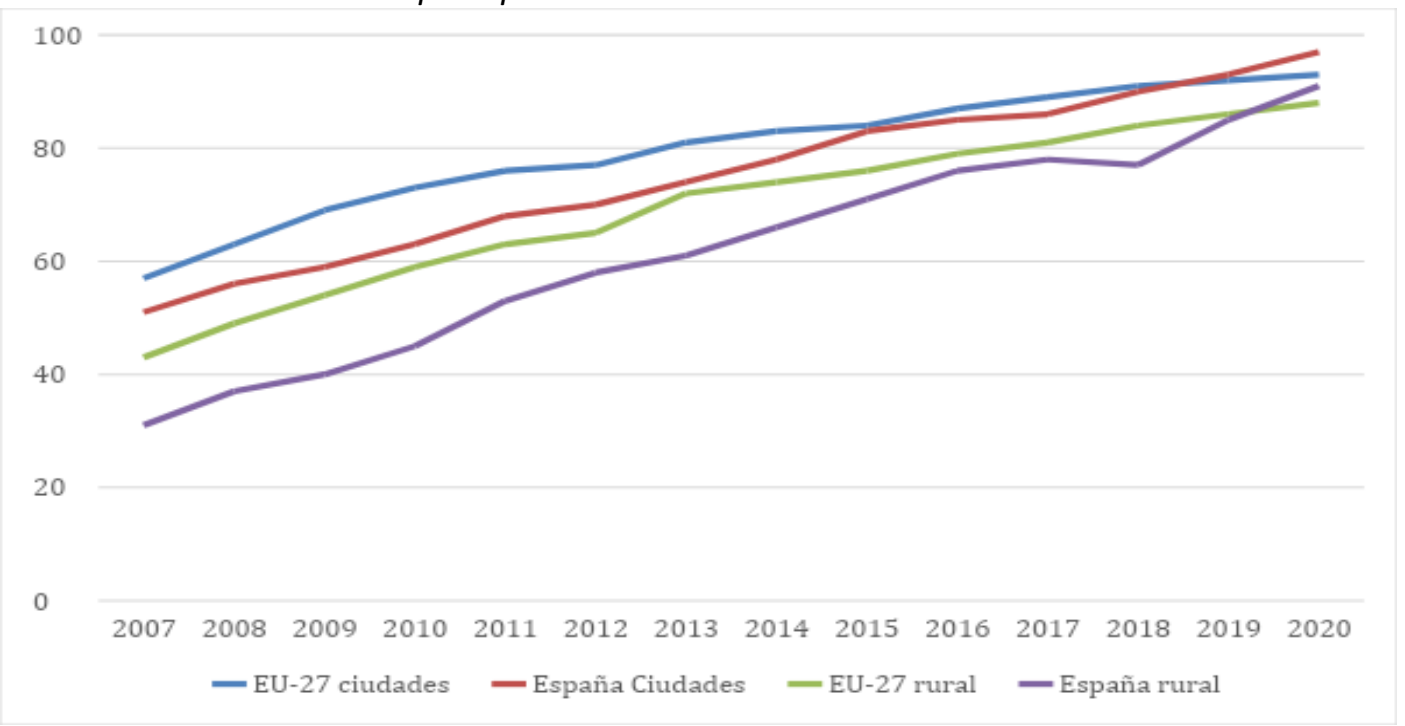

Fuente: Eurostat.

\footnotetext{
${ }^{13} \mathrm{Si}$ se pudiera analizar por comunidades autónomas, se verían diferencias entre ellas. Así se destaca en el texto de Carmona y Martínez (2007).
} 
En lo que respecta al argumento por el que no disponen de internet en casa, se aprecia que entre un $20 \%$ y un $30 \%$ de las personas que no tienen internet carecen de ello por el coste que supone el acceso, es decir el coste de los aparatos necesarios para el acceso.

No existen grandes diferencias entre los contextos, con la salvedad de las ciudades españolas en las que en 2018 el 28 \% de quienes no disponían de acceso a internet afirmaban que era por el coste, frente al $23 \%$ de los entornos rurales. En el contexto europeo no existen diferencias entre las ciudades y los entornos rurales.

GRÁFICA 2. Porcentaje de personas que no tienen internet por el coste del acceso. Respuesta entre las personas que no tienen internet. 2010 a 2020. Por nivel territorial.

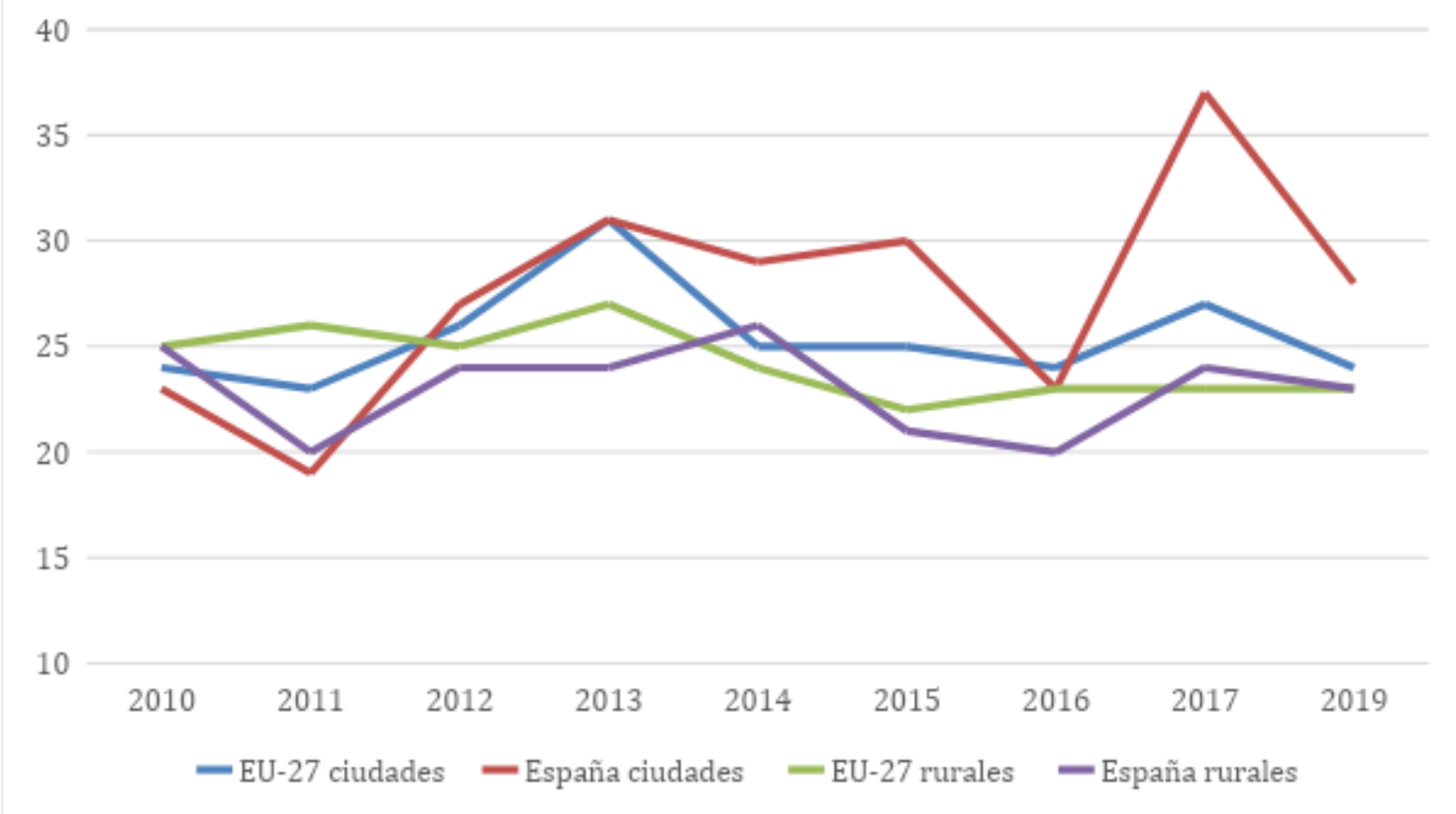

Fuente: Eurostat.

En lo que respecta a la falta de acceso a internet por parte de los hogares por motivos de coste del equipamiento, la cifra se incrementa en más de diez puntos porcentuales en todos los contextos salvo en el rural español, donde apenas si existe una variación de cinco puntos porcentuales. En los años de la crisis económica se incrementó esta justificación, sobre todo, en las ciudades, mientras que en el entorno rural esto no ocurrió, se mantuvo más estable. 
GRÁFICA 3. No tienen internet por el coste de los equipamientos. Respuesta entre las personas que no tienen internet. Por nivel territorial. 2011 a 2019.

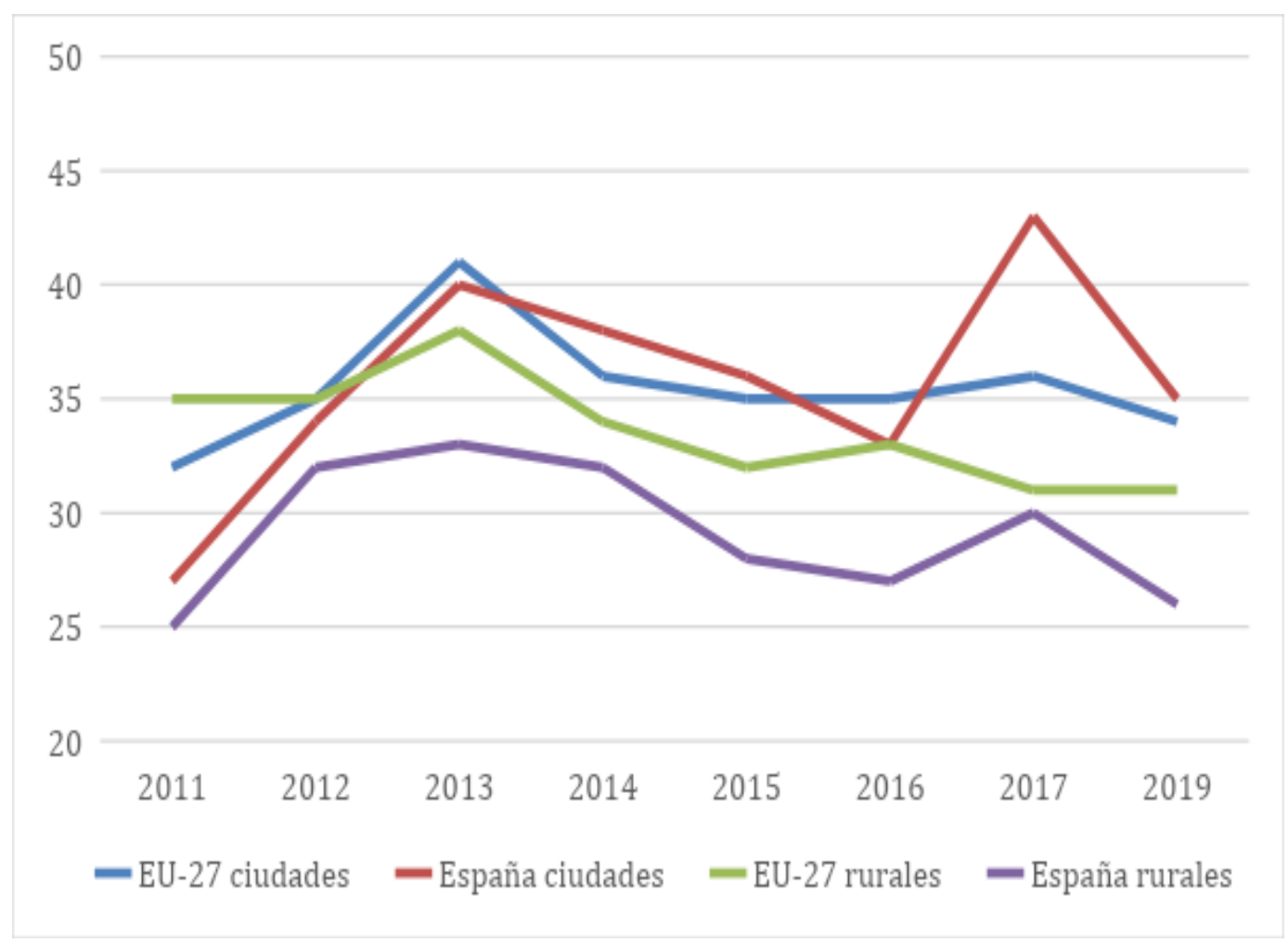

Fuente: Eurostat.

En la respuesta entre los que no tienen internet porque no les interesan los contenidos es donde existe la mayor diferencia entre el conjunto de Europa y España. En España, con independencia de si se trata de un contexto urbano o rural, el porcentaje de personas que señalaron que no les interesaban los contenidos de internet era superior a los de la media europea. El porcentaje de personas que afirmaban que no les interesaban los contenidos de internet se ha incrementado relativamente en España desde el año 2014. En este momento no se dispone de las cifras de este ítem para conocer lo que ha podido pasar como consecuencia de la pandemia. Entre las respuestas sobre por qué no se dispone de internet, esta es la mayoritaria, y la diferencia no parece entenderse dentro de la dicotomía rural-urbano, sino más bien desde los escenarios de los diversos países. 
GRÁFICA 4. No tienen internet porque no necesitan el acceso porque no lo necesitan. Respuesta entre las personas que no tienen internet. De 2010 a 2019. Por nivel territorial.

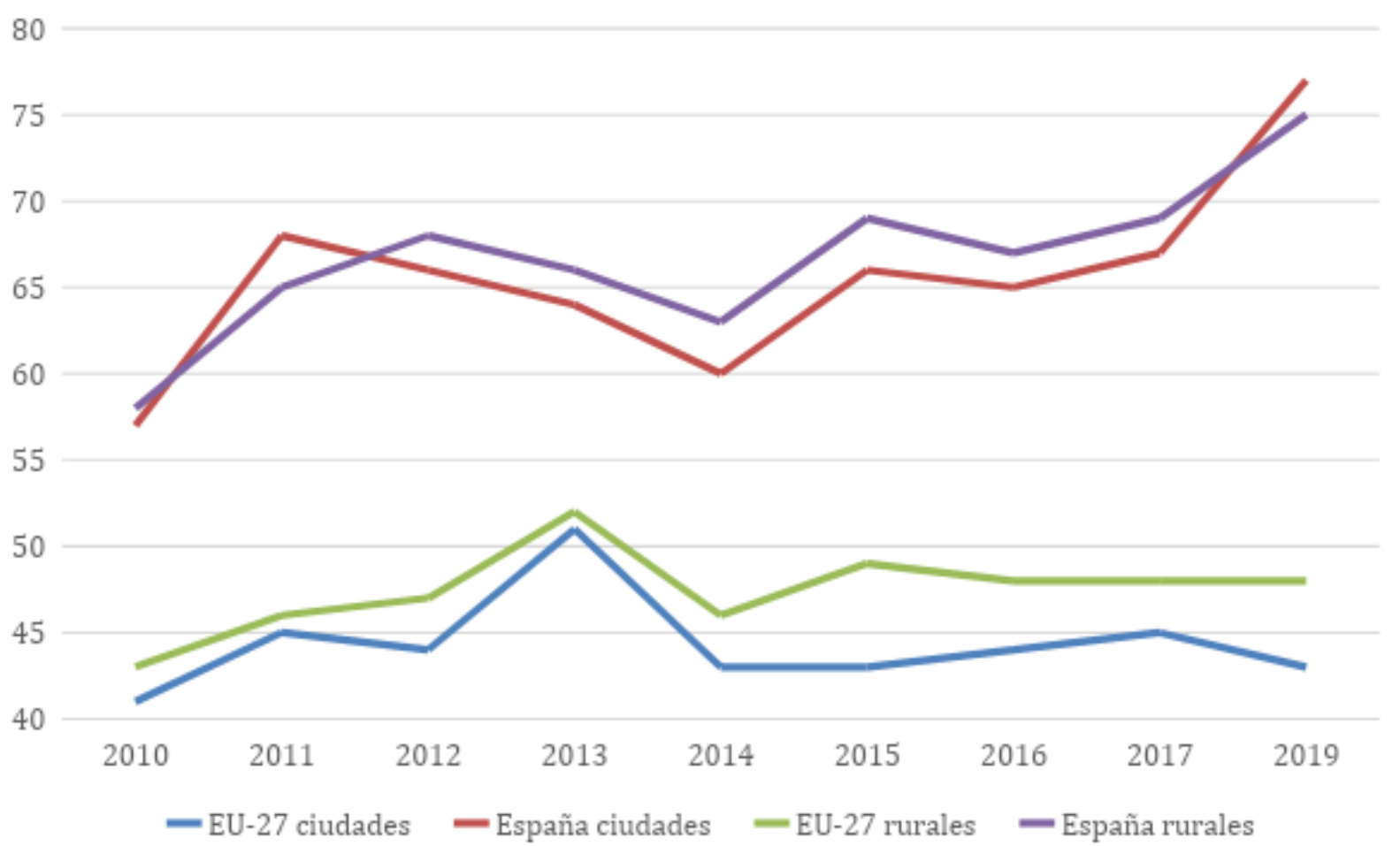

Fuente: Eurostat.

En lo que respecta al porcentaje de personas que nunca han usado internet en las zonas rurales, este es más elevado que en los contextos urbanos. Hace quince años el $50 \%$ de las personas residentes en los municipios pequeños no había usado nunca internet, y la diferencia con el contexto de las ciudades se situaba en torno a los veinte puntos. Así, en la dualidad rural la brecha se está reduciendo paulatinamente, pero todavía está presente.

En la encuesta de TIC se pregunta también por las capacidades con respecto a las nuevas tecnologías. En este sentido, las diferencias entre las personas que no tienen ninguna capacidad no es muy divergente que la que se refiere a los diferentes niveles de tamaño municipal. 
GRÁFICA 5. Porcentaje de personas que nunca han usado internet. De 2007 a 2020. Por nivel territorial.

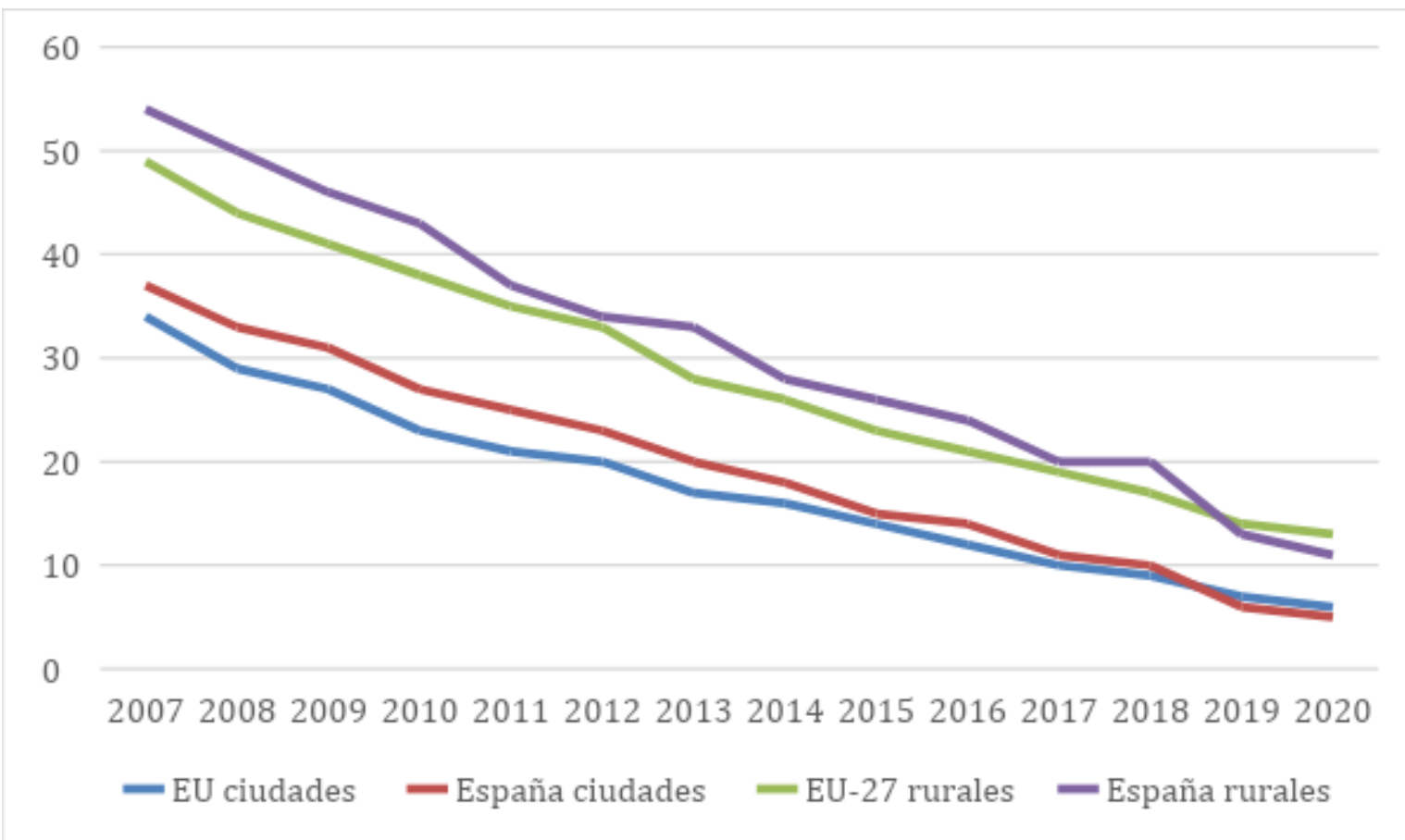

Fuente: Eurostat.

\section{CONCLUSIONES}

En este texto se han analizado las diferencias de contexto, es decir, se ha partido de la dicotomía rural-urbano sin atender a otras variables. Se considera también necesario un análisis en profundidad sobre ello atendiendo a las desigualdades de acceso que se dan por otras variables como, por ejemplo, la edad o el sexo.

En los últimos años, la diferencia en el acceso a las nuevas tecnologías se ha reducido en la dicotomía rural y urbano, incluso en algunas de las variables como al acceso a internet, las zonas rurales españolas se encuentran por encima de la media europea. Aun así, en Europa y en España en las zonas rurales el acceso a internet en menos que en las áreas rurales.

Hace apenas unos años el punto de partida en relación con las variables analizadas por parte de las personas que residían en los contextos rurales era muy diferente a la situación de las ciudades. En el contexto rural había un menor acceso a internet, un menor interés y una 
menor conexión. Mientras que en la actualidad esta situación ha cambiado, aún con las matizaciones que hemos analizado a lo largo del texto.

Las diferencias principales se han definido en términos de falta de interés por parte de algunas personas de los contextos rurales más que en cuestiones que tienen que ver con el coste o con el acceso. Además, esta es una tendencia que se ha incrementado en el último lustro, es decir, que se produce una desconexión voluntaria por una falta de interés en los contenidos, sobre todo en España.

El coste de internet y de los equipamientos es una de las razones por las que las personas manifestaron que no disponían de internet. En la dicotomía rural-urbano, la crisis económica fue un factor más decisivo para las ciudades que para los municipios de menor tamaño. Podríamos suponer que el mayor coste de la vida en las ciudades podría suponer una mayor pérdida de nivel adquisitivo ante dicha situación.

Bajo nuestro punto de vista, vemos que existe un comportamiento similar en el uso de las TICS en la UE y en España, pero resulta llamativo el hecho que en España un porcentaje elevado de personas que no tienen internet no disponen de él porque no lo necesitan. Este dato debe ser tenido en cuenta porque puede hablar del tipo de trabajos que se tienen el contexto y el futuro desarrollo del país.

A todo ello se suma que la población rural está más envejecida (en los dos contextos de referencia), y que a la vista de los datos existe un movimiento de personas en edad avanzada que se desplazan a los entornos rurales a su jubilación, movidos, quizás por la imagen de quietud y tranquilidad que se da en estas zonas.

\section{BIBLIOGRAFÍA}

BLANCO, Asunción; CANOVÉS, Gema. Teletrabajo, género y gentrificación en los espacios rurales: nuevos usos y nuevos protagonistas. Los casos de Cataluña y Ardèche (Francia). Geographicalia, 49: 99-110, 2006.

CARMONA, María Mercedes; GARCÍA, Leonarda. Difusión del uso de Internet en España. ¿Existe una brecha digital entre comunidades autónomas?. Revista de Estudios Regionales, 80: 193228. 2007.

CAMARERO, Luis. Alfonso (Coord.). La población rural de España: de los desequilibrios a la sostenibilidad social. La Caixa, Barcelona. 2009. 
COLOM, Antonio. Innovación organizacional y domesticación en Internet y las TIC en el mundo rural, con nuevas utilidades colectivas y sociales. La figura del Telecentro y en el Teletrabajo. Revista de economía pública, social y cooperativa, 49: 77-116, 2004.

EUROFOUND. Living, working and COVID-19, COVID-19 series, Publications Office of the European Union, Luxembourg, 2000.

FORONDA, Concepción. La integración de las Nuevas Tecnologías de la Información y Comunicación (NTIC) en el mundo rural. Sevilla: Universidad de Sevilla. Disponible en https://idus.us.es/handle/11441/64382., 2003.

GARNIER, Jean. Pierre. "Gentrification": un concepto inadecuado para una temática ambigua. Papeles de relaciones ecosociales y cambio global, 137: 13-26, 2017.

GONZÁLEZ, Antonio. La encuesta sobre equipamiento y uso de tecnologías de información y comunicación en los hogares (TIC-H) del INE. Índice. Revista de estadística y Sociedad, 55: 1012, 2013.

HART, L. Gary., LARSON, Eric. H. Y LISHER DENISSE. M. Rural definitions for health policy and research. American journal of public health, 95(7), 1149-1155, 2005.

HILDEBARD, Mireille. Smart technologies and the End(s) of Law. Cheltenhan, Edwar Elgar Publishing. 2016.

HOGGART, Keith. Not a definition of rural. Area. 20: 35-40, 1988.

JIMÉNEZ, Rocío. Ciudadanía digital y bienestar de las mujeres rurales en las redes sociales. RELATEC. Revista Latinoamericana de Tecnología Educativa, 15(2): 81-94, 2016

MARQUÉS, Pere. Usos educativos de Internet. ¿La revolución de la enseñanza. COMUNICACIÓN Y PEDAGOGÍA, 154: 37-44. 1998.

MILLER, Dorothy. A.. The 'sandwich' generation: adult children of the aging. Social Work, 26(5): 419-423, 1981.

TRPIN, Verónica. El desarrollo rural ante la nueva ruralidad. Algunos aportes desde los métodos cualitativos. AIBR, 42: 1-15, 2005.

SOROKIN, Pitirim A; ZIMMERMAN, Carle C. Principles of rural-urban sociology. Nueva York: Henry Holt, 1929.

Trabalho enviado em 13 de maio de 2021

Aceito em 04 de outubro de 2021 